\title{
Numerical Simulation and Experimental Research on Material Parameters Solution and Shape Control of Sandwich Panels with Aluminum Honeycomb
}

https://doi.org/10.1515/phys-2019-0057

Received Jun 16, 2019; accepted Aug 21, 2019

\begin{abstract}
This paper aims to solve two problems of the sandwich panel with aluminum honeycomb: material parameters solution and shape control. The accurate material parameters of the sandwich panels are the basis of shape control. Therefore, a mixed numerical-experimental method is proposed to inversely solve equivalent material parameters of the sandwich panel using genetic algorithm (GA) in the first place. Then a high efficiency FE model based on equivalent material parameters is established to study shape control of the sandwich panels. For shape control, the key issue aims to search optimum position and adjustment volume of control points where actuators are installed. Toward the end, the FE simulation method is deployed to optimize actuator position and adjustment volume one by one. Finally, an active control platform based on multi-point adjustment is developed to verify the practicability of the approach proposed in this paper. Through the experiment of shape control, the root mean square (RMS) of surface deviation of sandwich panel is decreased from $62.7 \mu \mathrm{m}$ to $15.5 \mu \mathrm{m}$. The results show that the shape control can significantly improve the surface accuracy of the sandwich panels, and the validity of equivalent material parameters is also proved from the side.
\end{abstract}

Keywords: sandwich panel; material parameters; genetic algorithm; shape control; finite element simulation

\footnotetext{
^Corresponding Author: Dongsheng Li: Beihang Univer-
sity,School of Mechanical Engineering \& Automation Beijing,

*Corresponding Author: Dongsheng Li: Beihang Univer-
sity,School of Mechanical Engineering \& Automation Beijing, 100083, China; Email: bhlidongs@126.com

Mingming Wang: Beihang University School of Mechanical Engineering \& Automation Beijing, 100083, China; Email: wmm704@buaa.edu.cn
}

Xianbin Zhou: Beihang University, School of Mechanical Engineering \& Automation Beijing, 100083, China; Email: xianbinzhou@163.com

Ә Open Access. (C) 2019 D. Li et al, published by De Gruyter. License
PACS: 02.50.Sk, 62.20.de, 62.20.dj, 87.55.de, 87.55.Gh

\section{Introduction}

The sandwich panels with aluminum honeycomb, for their advantages of light weight, high rigidity, great intensity and good electrical property, have been widely used in fabrication of the reflectors for microwave communication equipment, such as compact antenna test range (CATR), radio telescope, millimeter-wave radar, etc. The sandwich panels obtained by vacuum flexible forming proposed by professor ZHOU (Beihang University) have been successfully applied in fabrication of the reflectors for many CATRs [1-3]. Although these sandwich panels obtained from this forming process provides high-precision surface, it cannot satisfy high-precision requirements of the reflector panels when the working frequency is $110 \mathrm{GHz}$ or higher. According to American construction standards of the CATR, the RMS value of the surface deviation of reflector panels should be better than one percent of wave length corresponding to working frequency $[4,5]$. Therefore, it is very meaningful to study the method of further improving surface precision of the sandwich panels for expanding application scope and reducing scrap rate.

In recent years, a large number of investigations have focused on improving precision and controlling shape of the reflectors used in microwave communication equipment. Washington et al. [6, 7] investigated the optimization method of mechanically reconfigurable aperture antennas. A method termed as greatest error suppression method was proposed to optimize actuator position and actuation value. Theunissen et al. [8] developed a mechanical FE code that is incorporated into a diffraction synthesis code to enable direct synthesis of a reconfigurable dual offset reflector antenna contour beam in terms of the actuator amplitudes and positions. Ray M C. et al. $[9,10]$ conducted investigations on the active control of geomet- 

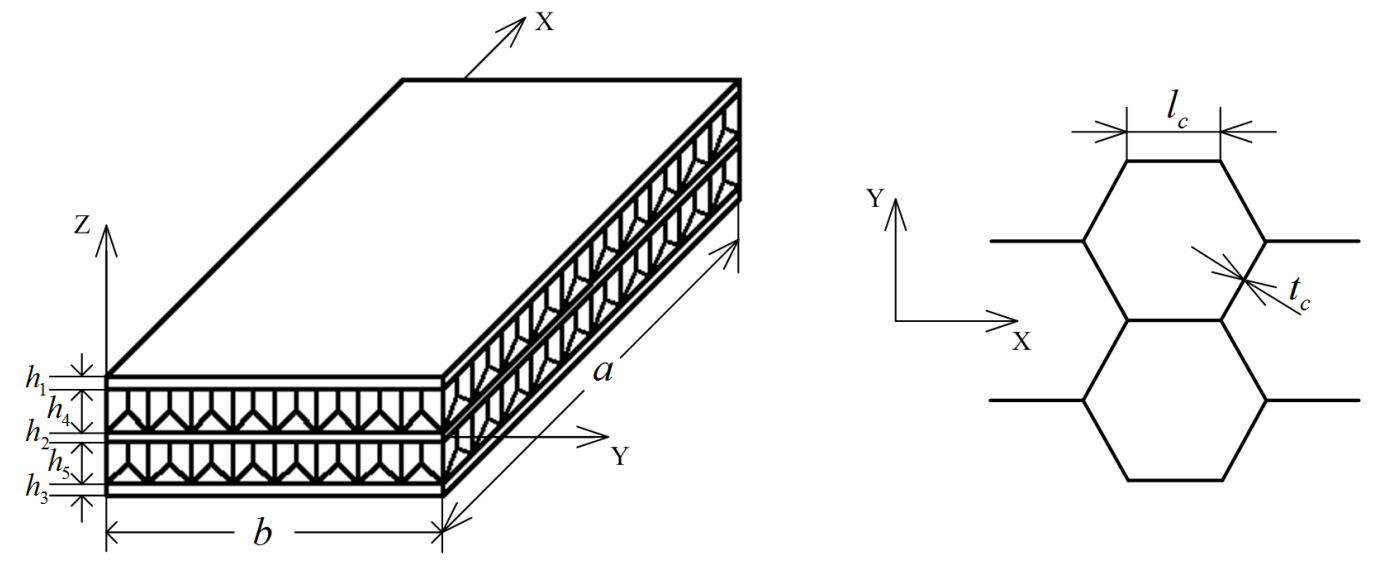

Figure 1: Geometric size of the sandwich panels with regular hexagonal honeycomb

rically nonlinear vibrations of doubly curved smart sandwich shells and functionally graded laminated composite shells using 1-3 piezoelectric composites. A three dimensional FE model was developed to study this coupled nonlinear electro-elastic problem. Wang et al. [11] established the FE model of the active structure with PZT actuator to adjust the shape profile of cable net structures. The optimal actuation voltages for the desired shape were evaluated by the quadratic criterion and min-max scheme respectively. The above approaches of shape control are more suitable to ameliorate surface precision of the panels fabricated by piezoelectric materials or weak rigidity plates. However, the sandwich panels involved in this study are made of ordinary aluminum plates and honeycombs, and this structure has a relatively high stiffness. It is very difficult to greatly improve and chronically ensure high-precision of the surface for sandwich panels just using the above method. Throughout these investigations, the key issues of shape control mainly involve two aspects: position optimization and adjustment optimization of the control points. Therefore, the FE method is deployed to optimize actuator position and adjustment volume for shape control of sandwich panels.

It is the first prerequisite to establish an accurate FE model of sandwich panels during FE analysis of shape control. Considering the computational efficiency, the honeycomb core must be equivalently modeled in actual engineering analysis. Wherefore, how to obtain effective material parameters plays an extremely important role in $\mathrm{FE}$ analysis of shape control. For similar materials, large numbers of investigations have focused on solving their parameters. A high-efficiency method of mixed numericalexperimental was proposed to estimate material parameters in 1990s [12, 13]. With the development of optimization algorithm and computer technology, the methodology is frequently employed to inversely seek mechanical and thermal properties of composite panels [14, 15], not only for homogeneous materials, but also anisotropic materials, such as laminated plates [16], sandwich panels [17, 18] and functional composite materials [19]. Based on the analysis of these researches, a mixed numerical-experimental method using GA will be investigated to evaluate material parameters of the sandwich panel with aluminum honeycomb.

As mentioned above, this study mainly focuses on two issues: material parameter solution of honeycomb cores and shape control of sandwich panels. Firstly, a mixed numerical-experimental method using GA is established to inversely solve equivalent material parameters of the sandwich panel based on stiffness experiment by fourpoint supporting and center loading. Then, the FE simulation method is deployed to optimize actuator position and adjustment volume for shape control of sandwich panels. The final meaningful work is to develop an active control platform based on multi-point adjustment to verify the practicability of the shape control for improving surface precision of the sandwich panel.

\section{Methodology Formulation}

\subsection{Equivalent $\mathrm{FE}$ model of sandwich panels}

The panel fabricated by vacuum flexible forming has a sandwich structure constituted of three-layer aluminum plates and two-layer aluminum honeycombs which are tightly spliced by enhanced epoxy glue [2]. Figure 1 shows the sketchy structure and key dimensions $\left(a, b, h_{1}, h_{2}\right.$, $h_{3}, h_{4}, h_{5}$ ) of the panel. According to the different anal- 
ysis objectives, there are many different equivalent modeling methods for sandwich panels with aluminum honeycomb. In order to improve the calculation efficiency, most of the finite element models of sandwich panels use two-dimensional shell units [20]. However, this modeling method cannot achieve the analysis requirements in this paper. In order to meet the analysis requirements of shape control, the equivalent FE model of sandwich panels is established based on sandwich panel theory. The three-layer aluminum plates are modeled as homogeneous isotropic shell with the same thickness as $h_{1}, h_{2}$ and $h_{3}$. The twolayer honeycomb cores are equivalent to a homogeneous orthotropic solid with the same thickness as $h_{4}$ and $h_{5}$. The aluminum plate is meshed by a quadrilateral shell element (S4R), and the honeycomb core is meshed by a threedimensional solid element (C3D8R) [21, 22], and the contact surface constraint type between the aluminum plate and the honeycomb core is tie. The equivalent FE model of the sandwich panel is shown in Figure 2.

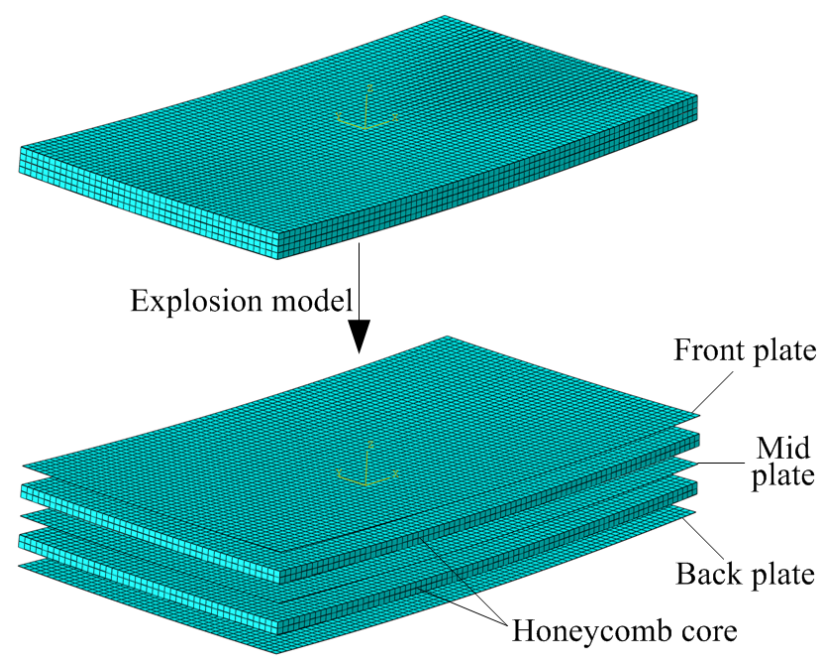

Figure 2: The equivalent FE model of the sandwich panels

\subsection{Inverse solution method of material parameters}

In the equivalent FE model of sandwich panels, the material parameters of the three-layer aluminum panels are obvious. However, the equivalent parameters of honeycomb core are relatively incomparable. In order to obtain these equivalent parameters, a mixed numerical-experimental method is proposed to inversely solve them using GA. Toward this end, stiffness experiment of sandwich panels is designed by four-point supporting and center loading.
Load zone, supporting position (S1 S4) and sample points (1 16) are shown in Figure 3. Due to the symmetry of the stiffness experiment, sample points are selected in a quarter area of the panel, and the choice of sixteen sample points is considered from the maximum deformation position to the minimum deformation position. Similarly, the corresponding FE analysis model of the stiffness experiment is established using the ABAQUS software. The deformation of the sample points can be respectively obtained by experiment and FE simulation. The deformation value obtained by the FE simulation can be made incomparably close to the experimental value by modifying the material parameters of the equivalent honeycomb core. The solution process is actually a Single-objective multivariate optimization problem. The GA is very suitable for solving this kind of problem, because of the advantage of global, robustness, universality and expandability.

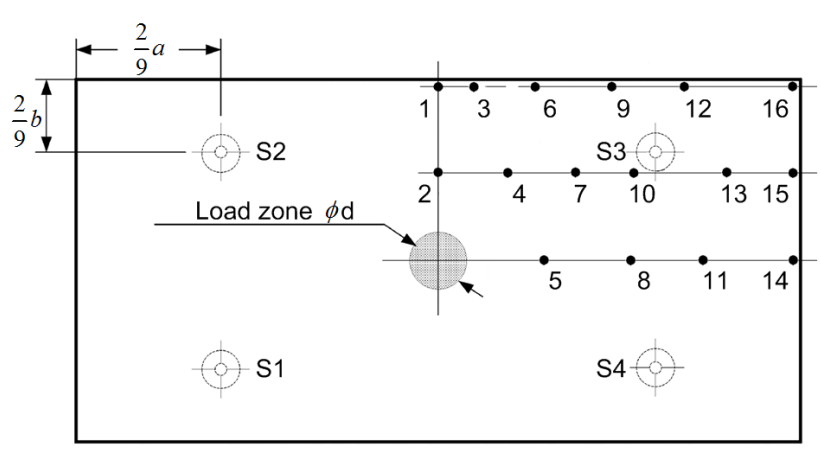

Figure 3: Load zone, support position and sample points of stiffness experiment

The effective Young's module, shear module and Poisson's ratio of the equivalent honeycomb core as optimization variables, as shown in equation (1).

$$
\begin{aligned}
& X= \\
& {\left[E_{c x}, E_{c y}, E_{c z}, G_{c x y}, G_{c x z}, G_{c y z}, \mu_{c x y}, \mu_{c x z}, \mu_{c y z}\right]}
\end{aligned}
$$

The experiment and simulation value of deformation at sample points are used to model the objective function $f(X)$ as:

$$
\min f(X)=\sqrt{\frac{1}{n} \sum_{i}^{n}\left(\delta_{i}-\delta_{i}^{\prime}\right)^{2}}
$$

Where $\delta_{i}$ is the experiment value of the deformation of the i-th sample point, $\delta_{i}^{\prime}$ is the simulation value of the deformation of corresponding sample points.

The optimization process is divided into forward analysis and inverse analysis. For the forward analysis, FE model is established for stiffness experiment of sandwich 


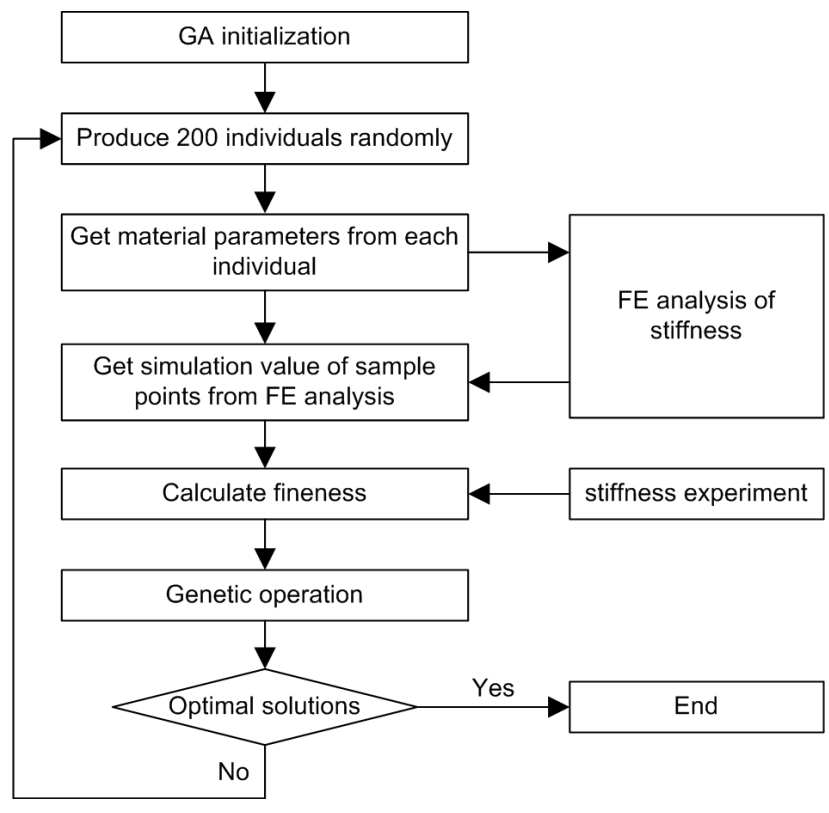

Figure 4: Inverse solution flow-chart for equivalent material parameters of honeycomb core

panels which the equivalent material parameters are estimated. For the inverse analysis, the GA method is employed to optimize and iterate the variable of material parameters according to the deviation between experiment value and simulation value. In order to improve the efficiency of parameter optimization, the GA method and FE analysis is seamlessly encapsulated by iSIGHT software. The optimization process of solution does not require human intervention, such as parameters modification, job creation, task submission, etc., which can greatly improve the efficiency of calculation. This routing optimization of reversing material parameters of this kind of sandwich panels is shown in Figure 4.

\subsection{Optimization method of shape control}

In this section, two optimization problems are solved: actuator position optimization and adjustment volume optimization. The geometric model truly reflecting actual error of the sandwich panel is firstly reconstructed as FE model according to the point-cloud data measured by laser tracker (Leica AT901-B). The maximum deviation will be chosen as corresponding position and adjustment of the first actuator. The comparison is done by imposing the displacement identical to the max error value on the reconstructed geometric model to force it into taking the shape of the designed CAD at the position with the max deviation. After the first simulation, the node coordinates on the sur-

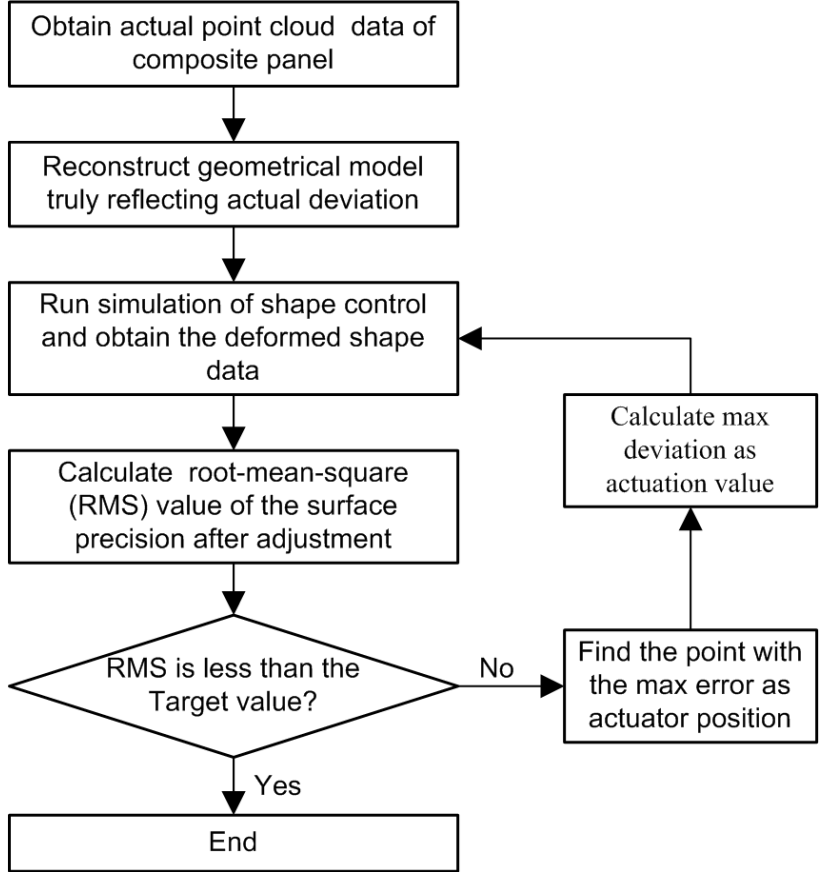

Figure 5: Optimization process flow-chart of shape control

face of the panel are output to a file. And then, the RMS value and maximum deviation of the adjusted surface are calculated according to the file. The aforementioned simulation process is repeated again and again until obtaining the desired RMS value of the surface error. The whole optimization process of the actuator position and actuation value is illustrated in Figure 5.

\section{Results and discussion}

\subsection{Equivalent material parameters}

The geometric size of the specimen used to solve the equivalent material parameters of the honeycomb core are followed as: $a=2450 \mathrm{~mm}, b=1305 \mathrm{~mm}, h_{1}=h_{3}=$ $1.5 \mathrm{~mm}, h_{2}=1.0 \mathrm{~mm}, h_{4}=h_{5}=48 \mathrm{~mm}, l_{c}=5.0 \mathrm{~mm}$ and $t_{c}=0.04 \mathrm{~mm}$.

The specification of the three-layer aluminum plate is YL12CZO, whose elastic modulus is 70000Mpa and poisson's ratio is 0.33 . The honeycomb cell is a regular hexagon with aluminum alloy 5052. Stiffness experiment based on four-point supporting and center loading is shown in Figure 6, and the deformation of sample points is measured by laser tracker. At the beginning of inversing equivalent materials, the initial value and feasible value range of the design variables are determined by theoretical explanation [23], as shown in Table 1. 
Table 1: Initial value and feasible domain of equivalent material properties

\begin{tabular}{cccccccccc}
\hline & \multicolumn{3}{c}{ Young's modulus/MPa } & \multicolumn{3}{c}{ Shear modulus / MPa } & \multicolumn{3}{c}{ Poisson ratio } \\
& $E_{c x}$ & $E_{c y}$ & $E_{c z}$ & $G_{c x y}$ & $G_{c x z}$ & $G_{c y z}$ & $u_{c x y}$ & $u_{c x z}$ & $u_{c y z}$ \\
\hline Initial value & 0.09 & 0.09 & 886.8 & 0.02 & 124.7 & 124.7 & 0.33 & - & - \\
Feasible domain & & $0 \sim 2000$ & & & $0 \sim 500$ & & & $0.1 \sim 0.5$ & \\
\hline
\end{tabular}

Table 2: Optimized equivalent material parameters of honeycomb core

\begin{tabular}{cccccccccc}
\hline & \multicolumn{3}{c}{ Young's modulus / $\mathrm{MPa}$} & \multicolumn{3}{c}{ Shear modulus / MPa } & \multicolumn{3}{c}{ Poisson ratio } \\
& $E_{c x}$ & $E_{c y}$ & $E_{c z}$ & $G_{c x y}$ & $G_{c x z}$ & $G_{c y z}$ & $u_{c x y}$ & $u_{c x z}$ & $u_{c y z}$ \\
\hline Equivalent value & 0.136 & 0.136 & 975.5 & 0.06 & 118.47 & 118.47 & 0.34 & 0 & 0 \\
\hline
\end{tabular}

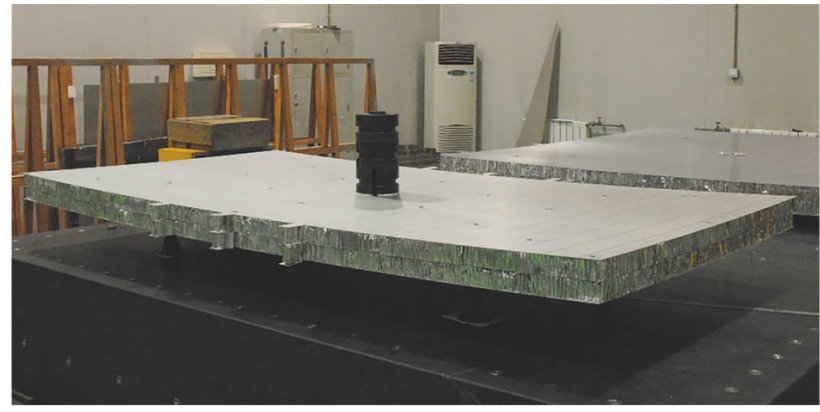

Figure 6: Stiffness experiment of the sandwich panels

The exactly equivalent material parameters of the panel are obtained through the optimization process shown in Figure 4. During the inverse seeking, elitist strategy is adopted, the mutation rate is set at $1 \%$ and the crossover rate is set at $8 \%$. The convergence history of the synthesis algorithm is displayed in Figure 7. It is evident that the optimal solution is obtained when the GA procedures run for 1000 generations with objection better than $2.9 \mathrm{um}$. At this time, the equivalent material parameters of honeycomb core are optimized and solved through mixed numerical-experimental method, as listed in Table 2. In order to prove the reliability of equivalent parameters, experiment and simulation results are separately compared under bearing load $100 \mathrm{~N}, 200 \mathrm{~N}, 300 \mathrm{~N}$ and $400 \mathrm{~N}$. The comparison curves between experiment and simulation results are respectively drawn in Figure 8. As known from Figure 8 , the dispersion between the simulated and experimental values is larger at low loads. A possible reason is that there is a system error in the stiffness experiment, such as measurement uncertainty. The deformation amount of the sample points increases with the load increasing, however the system error may be constant. The ratio of the system error to the deformation is decreasing with the load increasing. Excepting this situation, the consistent between experiment and simulation is quite high. The stiffness ex-

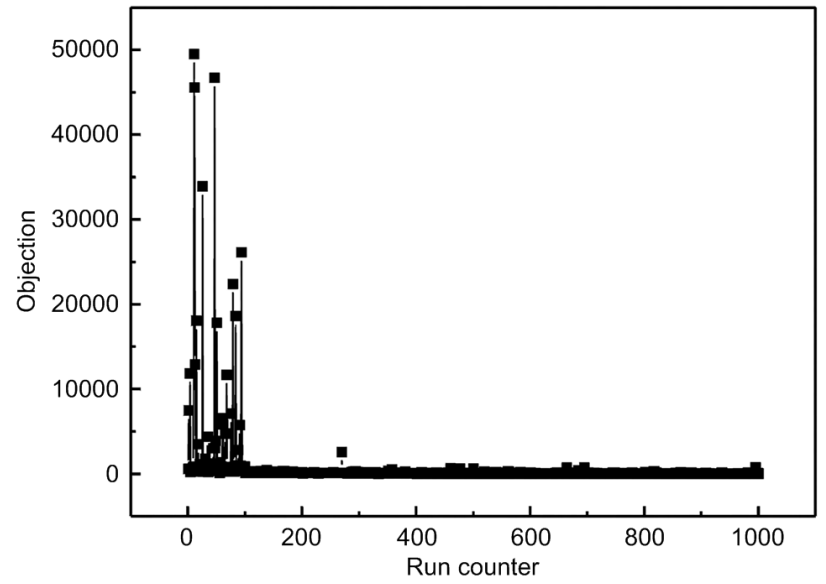

Figure 7: Convergence history of material parameters reverse solution using $\mathrm{GA}$

periment results show that the equivalent model of the sandwich panel is effective, and the solution of the equivalent material parameters of the honeycomb core is accurate.

\subsection{Shape control of sandwich panels}

On the basis of equivalent material model, FE Analysis example of shape control for the sandwich panel is carried out. The experiment piece used for the shape control has the same geometry as that of stiffness test. The deviation between the actual surface and the theoretical surface is measured and calculated by laser tracker system (Leica AT901-B). The RMS of surface deviation is $0.0627 \mathrm{~mm}$ with max error $0.178 \mathrm{~mm}$ and min error- $0.142 \mathrm{~mm}$, as shown in Figure 9.

In the first place, FE simulation of shape control process for the specimen is carried out. From Figure9 it can be found that the initial maximum deviation occurs at the 

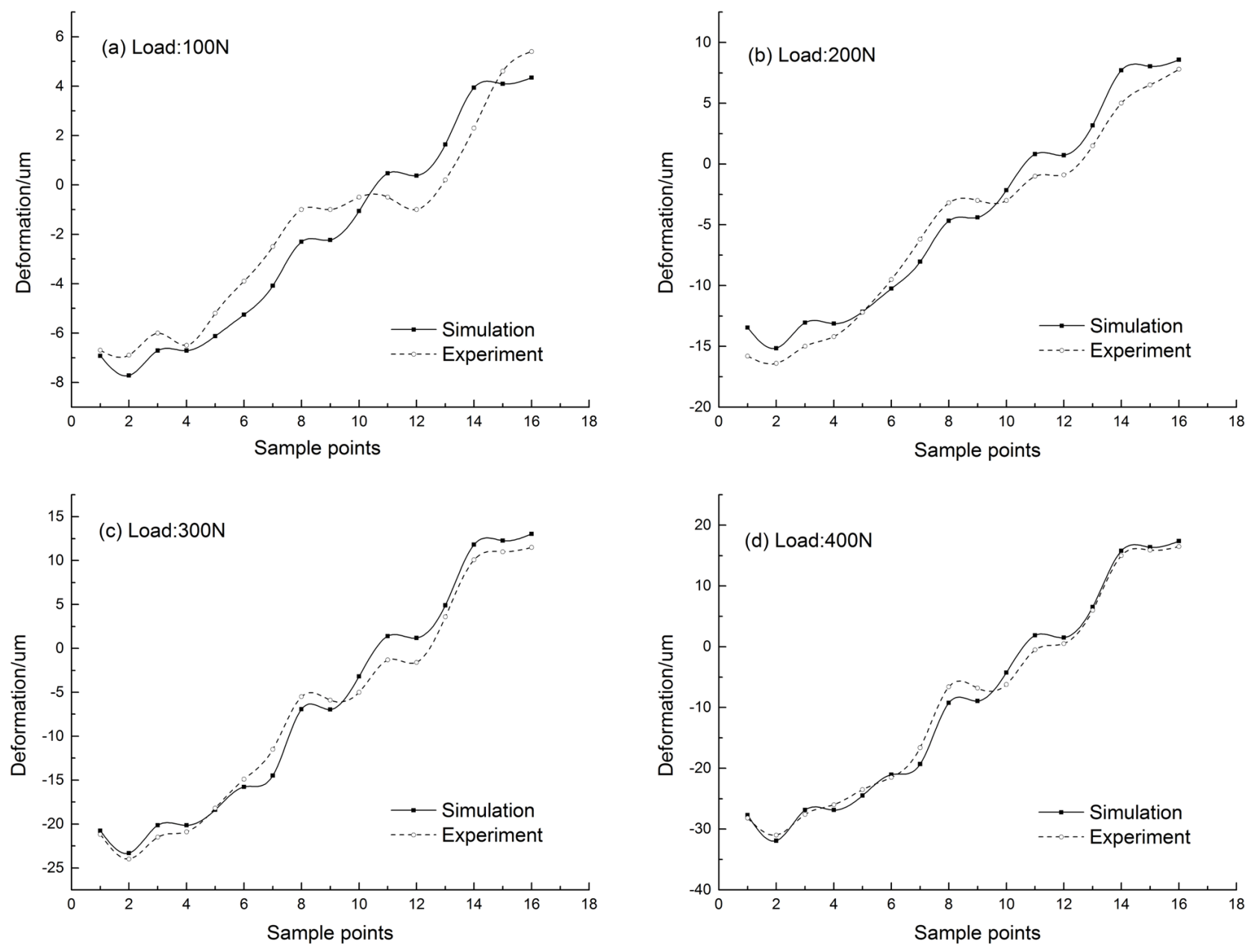

Figure 8: Results comparison between experiment and simulation bearing load 100N (a), 200N (b), 300N (c) and 400N (b)

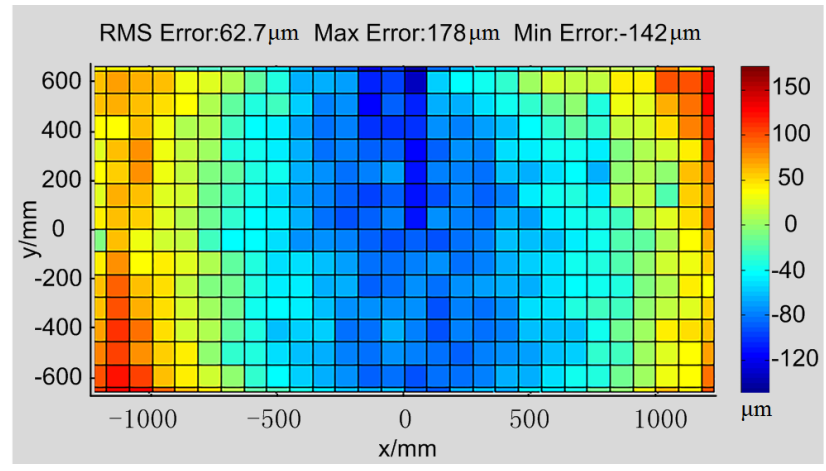

Figure 9: Surface deviation distribution of experimental piece

four corners of the sandwich panel. Therefore, the first simulation of the shape control for the panel is done by imposing respective displacement on the four corners of the model. After the first adjustment, the surface precision (RMS value) of the panel is improved from $0.0627 \mathrm{~mm}$ to $0.038 \mathrm{~mm}$, and the peak-to-peak value of the deviation is ameliorated from $0.320 \mathrm{~mm}$ to $0.138 \mathrm{~mm}$. The actuator position and error distribution of the panel surface after the first adjustment is shown in Figure 10.

Although the surface precision of the panel has been greatly improved through the first adjustment, it still does not meet the target precision. And therefore, another adjustment is needed. The second adjustment is conducted under the results of the first simulation. The four control points of the first regulation should be set as fixed constraint along z-direction of the panel. The second simulation is executed by imposing actuator value at the middle position of the two long sides. The RMS and peak-topeak value of the panel respectively reach to $0.024 \mathrm{~mm}$ and $0.108 \mathrm{~mm}$. Figure 11 displays the position of the actuator and corresponding error distribution of the surface after the second adjustment. Figure 12 displays the position of the actuator and corresponding error distribution of the surface after the third adjustment. This simulation process is repeated until the surface precision achieves the 

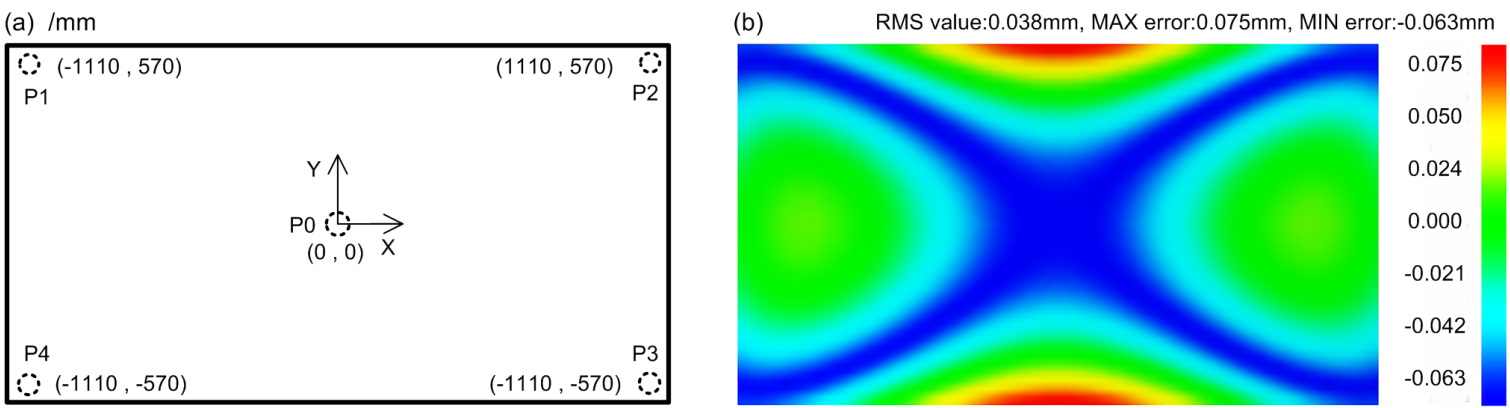

Figure 10: Actuator position (a) and error distribution (b) of the sandwich panel after first adjustment
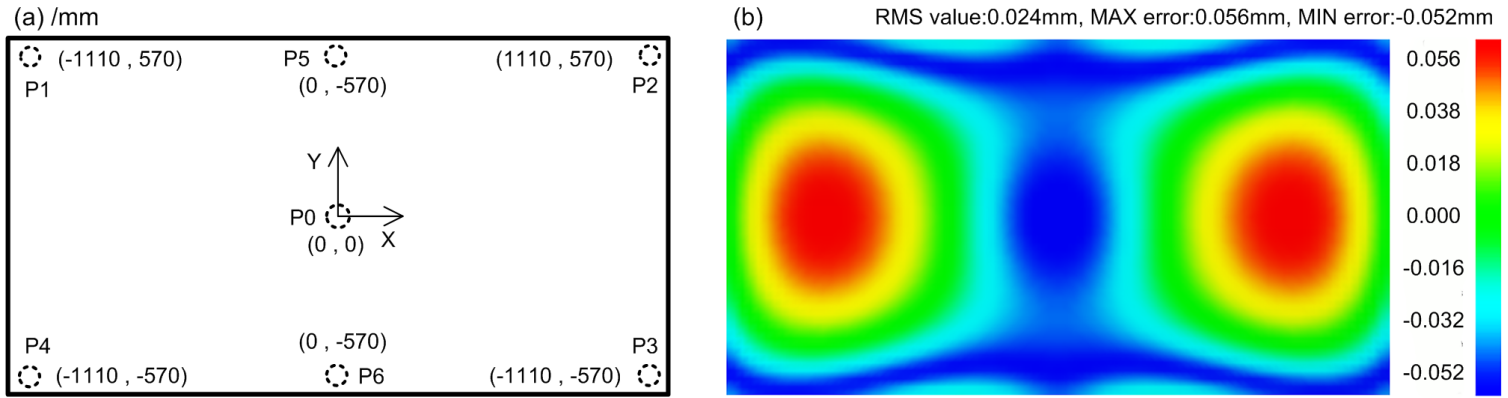

Figure 11: Actuator position (a) and error distribution (b) of the sandwich panel after second adjustment
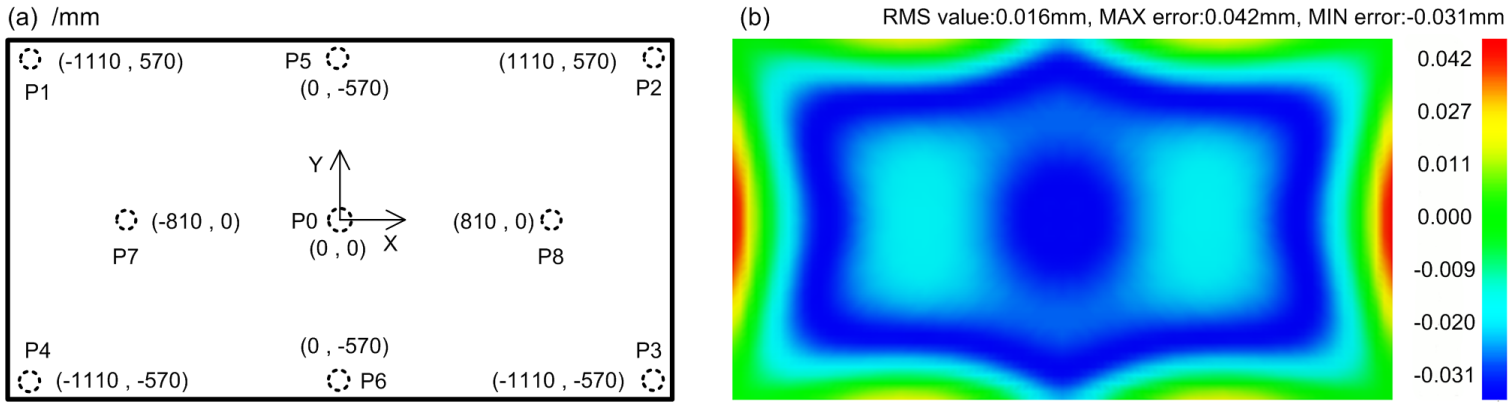

Figure 12: Actuator position (a) and error distribution (b) of the sandwich panel after third adjustment
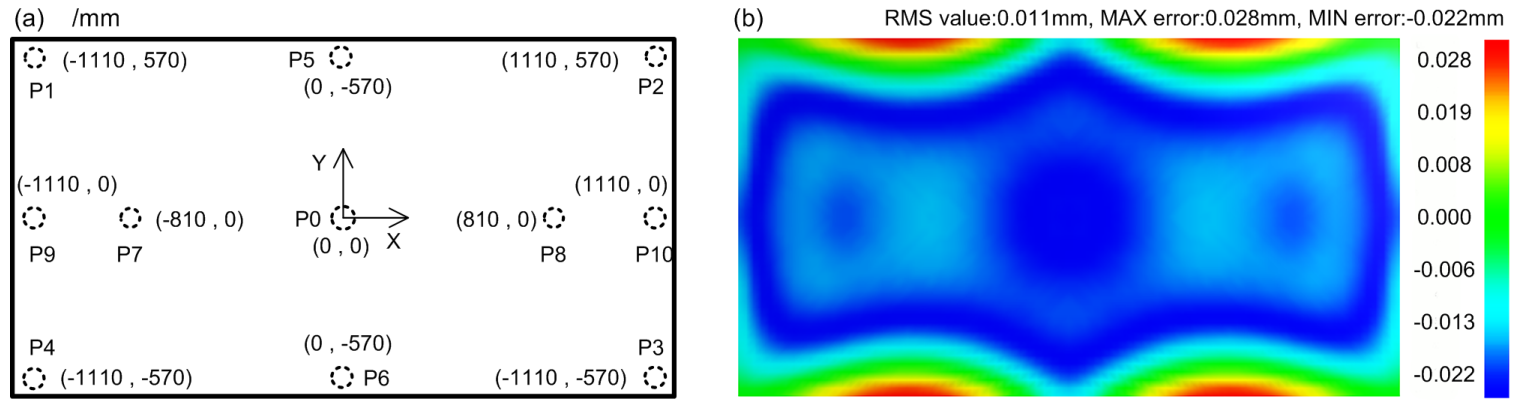

Figure 13: Actuator position (a) and error distribution (b) of the sandwich panel after final adjustment

required accuracy of the panel. Finally, the surface precision of the panel comes up to $0.011 \mathrm{~mm}$ through four times adjustment. Ultimate actuator position and deviation distribution of the panel are shown in Figure 13.
Through comprehensively analyzing the results of above simulation, eleven actuators are necessary to improve the surface precision of the sandwich panel with deviation caused by insufficient spring-back. The actua- 
Table 3: Actuator position and value obtained by FE simulation

\begin{tabular}{cccc}
\hline $\begin{array}{c}\text { Actuator } \\
\text { N0. }\end{array}$ & $\begin{array}{c}\text { Actuator } \\
\text { value } / \mathrm{mm}\end{array}$ & $\begin{array}{c}\text { Actuator } \\
\text { NO. }\end{array}$ & $\begin{array}{c}\text { Actuator } \\
\text { value } / \mathrm{mm}\end{array}$ \\
\hline P0 & 0.116 & P6 & 0.109 \\
P1 & -0.095 & P7 & 0.032 \\
P2 & -0.149 & P8 & 0.054 \\
P3 & -0.075 & P9 & -0.060 \\
P4 & -0.164 & P10 & -0.085 \\
P5 & 0.138 & - & - \\
\hline
\end{tabular}

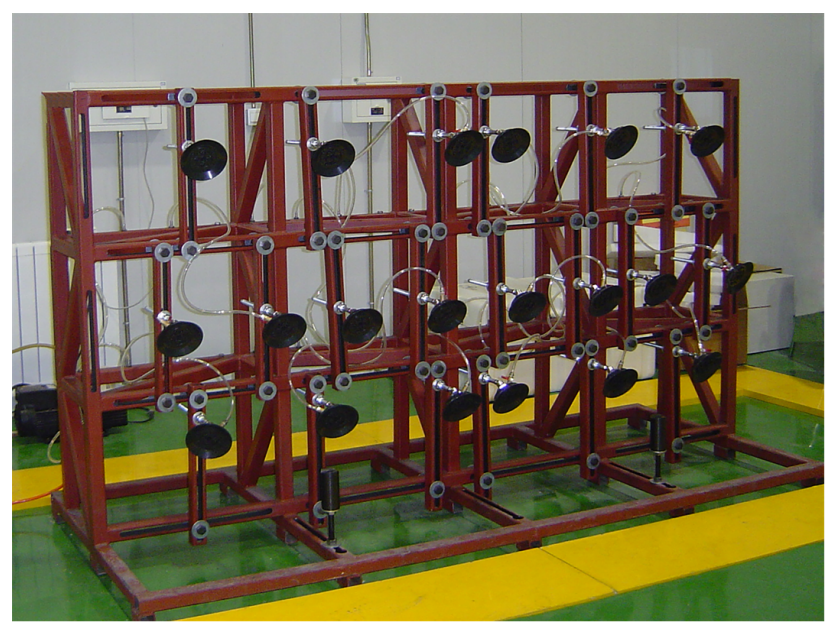

Figure 14: Substance of the back support structure

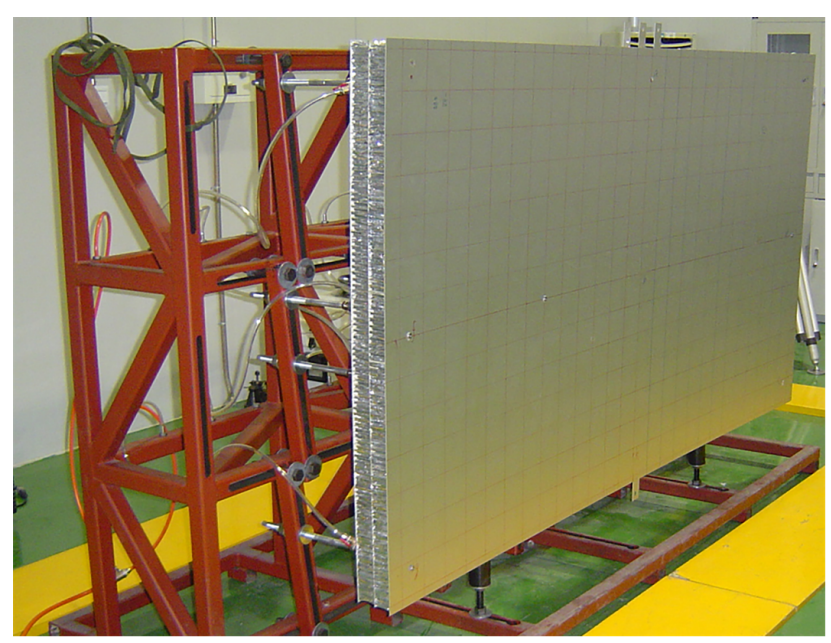

Figure 15: Adjustment experiment of shape control

tion values are calculated by four-point-constrain leastsquares method, as depicted in Table 3.

In order to verify the effectiveness of the shape control method proposed in this paper, a test platform based on multi-point adjustment is established. The platform mainly consists of back support structure and vacuum system, as shown in Figure 14. The actuator is movable along

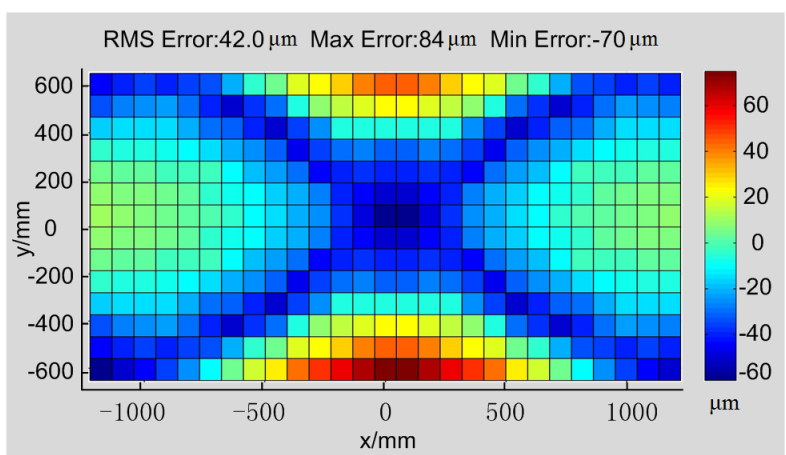

(a) First shape control experiment

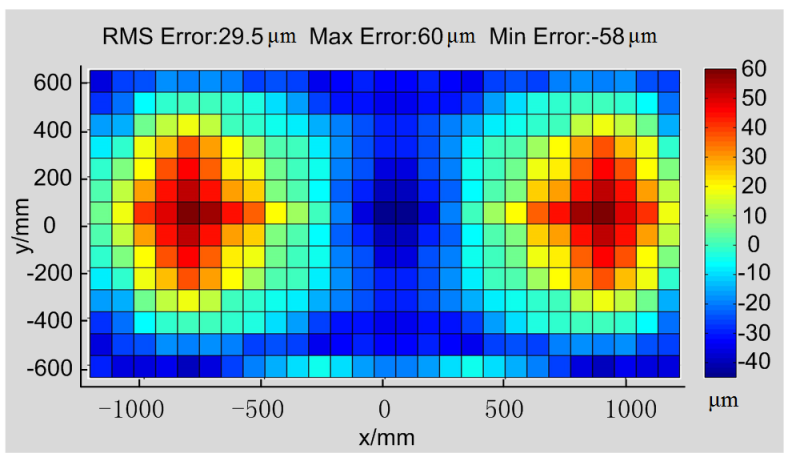

(b) Second shape control experiment

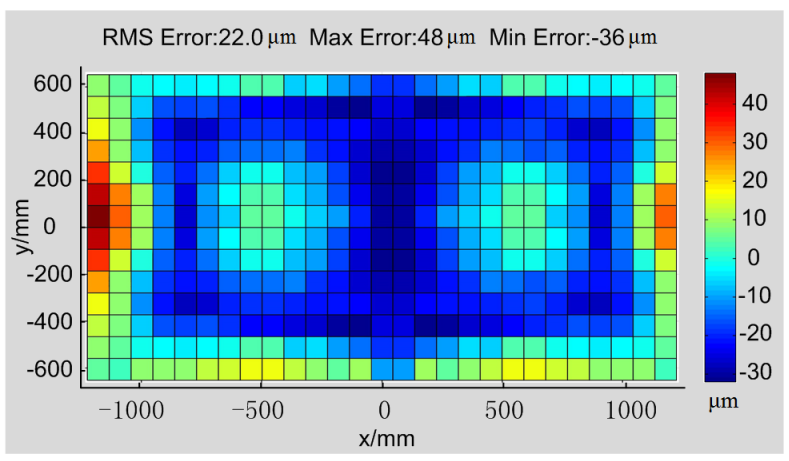

(c) Third shape control experiment

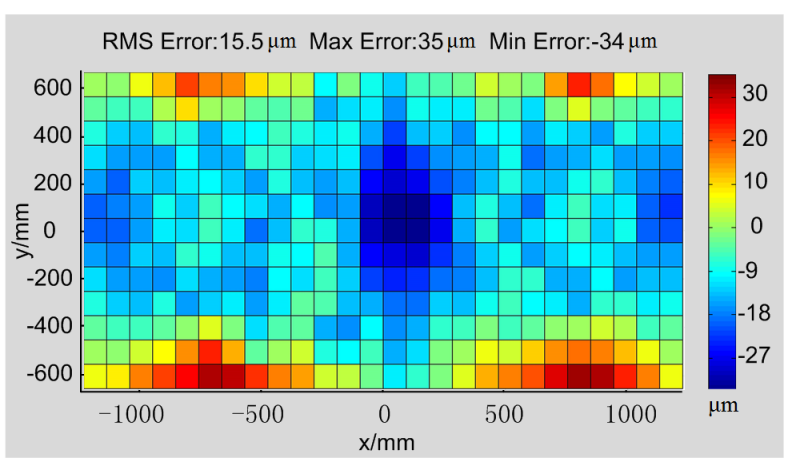

(d) Fourth shape control experiment

Figure 16: Error distribution of the test panel through shape control 
$\mathrm{x}$-direction and y-direction, and the adjustment along zdirection is performed through screw driver, and the panel is bonded to the back structure by vacuum chuck.

The sandwich panel is mounted on the back structure by the way shown in Figure 15. Before that, these actuators required are installed on the back support structure according to the position coordinates obtained by simulation. Eleven actuators required are set according to the position coordinates shown in Figure 13a, and the corresponding actuation value listed in Table 3 is imposed on the panel one by one. According to the FE optimization process of shape control,five actuators (P0, P1, P2, P3, P4) are applied to the panel with the actuation value in Table 3 in the first. After the first adjustment, surface deviation of the panel is decreased from $62.7 \mu \mathrm{m}$ to $42.0 \mu \mathrm{m}$, and the error distribution is shown in Figure 16(a). The whole surface deviation of the panel is redistributed after five actuators are added. As known from comparing Figure 16(a) and $10(\mathrm{~b})$, the deformation trend of simulation and experiment is substantially unanimous. Then two actuators (P5, P6) are added to the panel again, and the surface accuracy reaches $29.5 \mu \mathrm{m}$, as shown in Figure 16(b). The surface accuracy reaches $22 \mu \mathrm{m}$ after two more actuators (P7, P8), as shown in Figure 16(c). Finally, P9 and P10 actuators are applied to complete the fourth adjustment. Figure 16(d) displays the final error nephogram after shape adjustment test, with the RMS value $15.5 \mu \mathrm{m}$ and peak-topeak value $69 \mu \mathrm{m}$. Through shape control, surface accuracy of the sandwich panel increased by $75 \%$ (RMS). The results show that the method of shape control based on multi-point adjustment is effective to improve the surface precision of the sandwich panel. From the other hand, the results also show that the solution of the equivalent material parameters of honeycomb core is accurate. Comparing experiment results and simulation results of shape control, the consistency between experiments and simulations of the shape control is good. This result indicates that actuator position and adjustment value obtained by FE simulation can be used to guide the actual production. Number and position of the necessary actuators should be determined according to the accuracy requirements of the reflector panels.

\section{Conclusion}

The equivalent FE model of sandwich panels with honeycomb core is established based on sandwich panel theory. The honeycomb core is equivalent to a homogeneous orthotropic layer with uniform thickness. A mixed numerical-experimental method combining stiffness experiment and FE simulation with four-point support and center loading is designed to inversely solve the equivalent material parameters of the honeycomb. The optimal equivalent parameters of the honeycomb core are obtained by GA. The experiment and FE simulation under bearing load $100 \mathrm{~N}, 200 \mathrm{~N}, 300 \mathrm{~N}$ and $400 \mathrm{~N}$ are carried out to prove the reliability of equivalent parameters. The results show that the equivalent model of the sandwich panel is effective, and the solution of the equivalent material parameters of the honeycomb core is accurate.

On the basis of equivalent FE model, the FE analysis method of shape control is developed to optimize actuator position and adjustment volume. The shape control method and experiment platform based on multi-point adjustment is established to improve the surface precision of the sandwich panel. Through shape control, the sandwich panel with initial surface deviation $62.7 \mu \mathrm{m}$ (RMS) is decreased to $15.5 \mu \mathrm{m}$ (RMS). The results indicate that shape control based on multi-point adjustment is higher efficiency and better adaptability to improve shape precision for sandwich panels. On the other hand, the result also shows that the solution of the equivalent material parameters of honeycomb core is accurate.

Acknowledgement: The work was supported by the National Natural Science Foundation of china (grant nos. 51575028). The authors gratefully thank Hesheng CHANG and Changling HAO for their substantial and meaningful work.

\section{References}

[1] Wang M., Li D., Zhou X., and He G., Design, fabrication and onsite alignment of low-cost reflector used in large-scale compact antenna test range, 2017 11th Eur. Conf. Antennas Propagation, EUCAP 2017, 2017, 2590-2594.

[2] Changling H., Xianbin Z., and Xiaoqiang L., Solution to Boundaryvalue Problems in Fabrication of High-precision Reflector Panels, Chinese J. Aeronaut, 2009, 22, 97-104

[3] Zhou G., Li X., Li D., Luan J., and Zhao J., Surface accuracy of a large-scale compact antenna test range considering mechanism, metrology and alignment, Meas. Sci. Technol., 2014, 25.

[4] Ruze J., Antenna Tolerance Theory-A Review, Proc. IEEE, 1966, 54, 633-640.

[5] Rahmat-Samii Y., An Efficient Computational Method for Characterizing the Effects of Random Surface Errors on the Average Power Pattern of Reflectors, IEEE Trans. Antennas. Propag., 1983, 31, 92-98.

[6] Washington G., Yoon H.S., and Angelino M., Theunissen WH. Design, modeling, and optimization of mechanically reconfigurable aperture antennas, IEEE Trans. Antennas. Propag., 2002, 
$50,628-637$.

[7] Yoon H.S., and Washington G., An optimal method of shape control for deformable structures with an application to a mechanically reconfigurable reflector antenna, Smart Mater Struct., 2010, 19, 105004.

[8] Theunissen W.H., Yoon H.T., Burnside W.D., and Washington G.N., Reconfigurable contour beam-reflector antenna synthesis using a mechanical finite-element description of the adjustable surface. IEEE Trans. Antennas Propag., 2001, 49, 272-279.

[9] Sarangi S.K., and Ray M.C., Smart control of nonlinear vibrations of doubly curved functionally graded laminated composite shells under a thermal environment using 1-3 piezoelectric composites, Int. J. Mech. Mater. Des., 2013, 9, 253-280.

[10] Suresh Kumar R., and Ray M.C., Active control of geometrically nonlinear vibrations of doubly curved smart sandwich shells using 1-3 piezoelectric composites, Compos. Struct., 2013, 105, 173-187.

[11] Wang Z., Li T., and Cao Y., Active shape adjustment of cable net structures with PZT actuators, Aerosp. Sci. Technol., 2013, 26, 160-168.

[12] Frederiksen P.S., Identification of temperature dependence for orthotropic material moduli, Mech. Mater., 1992, 13, 79-90.

[13] Setiawan R., Syngellakis S., and Hill M., A metamodeling approach to mechanical characterization of anisotropic plates, J. Compos. Mater., 2009, 43, 2333-2349.

[14] Monteau J.Y., Estimation of thermal conductivity of sandwich bread using an inverse method. J. Food Eng. 2008, 85, 132-140.

[15] Liu G.R., Lee J.H., Patera A.T., Yang Z.L. and Lam K.Y., Inverse identification of thermal parameters using reduced-basis method, Comput. Methods Appl. Mech. Eng., 2005, 194, 3090-3107.
[16] Matter M., Gmür T., Cugnoni J., and Schorderet A., Improved modal characterization of the constitutive parameters in multilayered plates, Compos. Sci. Technol., 2007, 67, 1121-1131.

[17] Shi Y., Sol H., and Hua H., Material parameter identification of sandwich beams by an inverse method, J. Sound Vib., 2006, 290, 1234-1255.

[18] Bezazi A., Remillat C., Innocenti P., and Scarpa F., In-plane mechanical and thermal conductivity properties of a rectangularhexagonal honeycomb structure, Compos. Struct., 2008, 84, 248-255.

[19] Araújo A.L., Mota Soares C.M., Herskovits J. and Pedersen P., Development of a finite element model for the identification of mechanical and piezoelectric properties through gradient optimisation and experimental vibration data, Compos. Struct., 2002, 58, 307-318.

[20] Hu H., Belouettar S., Potier-Ferry M., and Daya E.M., Review and assessment of various theories for modeling sandwich composites, Compos. Struct., 2008, 84, 282-292.

[21] Shanmugam N.E., Kumar G., and Thevendran V., Finite element modelling of double skin composite slabs, Finite Elem. Anal. Des., 2002, 38, 579-599.

[22] Rahman H., Jamshed R., Hameed H., and Raza S., Finite Element Analysis (FEA) of Honeycomb Sandwich Panel for Continuum Properties Evaluation and Core Height Influence on the Dynamic Behavior, Adv. Mater. Res., 2011, 326, 1-10.

[23] Masters I.G., and Evans K.E., Models for the elastic deformation of honeycombs, Compos. Struct., 1996, 35, 403-422. 